\title{
Estimating Frequency Moments of Data Streams using Random Linear Combinations
}

\author{
Sumit Ganguly \\ Indian Institute of Technology, Kanpur \\ e-mail: sganguly@iitk.ac.in
}

\begin{abstract}
The problem of estimating the $k^{t h}$ frequency moment $F_{k}$ for any nonnegative $k$, over a data stream by looking at the items exactly once as they arrive, was considered in a seminal paper by Alon, Matias and Szegedy $[1,2]$. The space complexity of their algorithm is $\tilde{O}\left(n^{1-\frac{1}{k}}\right)$. For $k>2$, their technique does not apply to data streams with arbitrary insertions and deletions. In this paper, we present an algorithm for estimating $F_{k}$ for $k>2$, over general update streams whose space complexity is $\tilde{O}\left(n^{1-\frac{1}{k-1}}\right)$ and time complexity of processing each stream update is $\tilde{O}(1)$.

Recently, an algorithm for estimating $F_{k}$ over general update streams with similar space complexity has been published by Coppersmith and Kumar [7]. Our technique is, (a) basically different from the technique used by [7], (b) is simpler and symmetric, and, (c) is significantly more efficient in terms of the time required to process a stream update $\left(\tilde{O}(1)\right.$ compared with $\left.\tilde{O}\left(n^{1-\frac{1}{k-1}}\right)\right)$.
\end{abstract}

\section{Introduction}

A data stream can be viewed as a sequence of updates, that is, insertions and deletions of items. Each update is of the form $(l, \pm v)$, where, $l$ is the identity of the item and $v$ is the change in frequency of $l$ such that $|v| \geq 1$. The items are assumed to draw their identities from the domain $[N]=\{0,1, \ldots, N-1\}$. If $v$ is positive, then the operation is an insertion operation, otherwise, the operation is a deletion operation. The frequency of an item with identity $l$, denoted by $f_{l}$, is the sum of the changes in frequencies of $l$ from the start of the stream. In this paper, we are interested in computing the $k^{\text {th }}$ frequency moment $F_{k}=\sum_{l} f_{l}^{k}$, for $k>2$ and $k$ integral, by looking at the items exactly once when they arrive.

The problem of estimating frequency moments over data streams using randomized algorithms was first studied in a seminal paper by Alon, Matias and Szegedy [1,2]. They present an algorithm, based on sampling, for estimating $F_{k}$, for $k \geq 2$, to within any specified approximation factor $\epsilon$ and with confidence that is a constant greater than $1 / 2$. The space complexity of this algorithm is $s=\tilde{O}\left(n^{1-\frac{1}{k}}\right)$ (suppressing the term $\left.\frac{1}{\epsilon^{2}}\right)$ and time complexity per update is $\tilde{O}\left(n^{1-\frac{1}{k}}\right)$, where, $n$ is the number of distinct elements in the stream. This algorithm assumes that frequency updates are restricted to the form $(l,+1)$.

One problem with the sampling algorithm of $[1,2]$ is that it is not applicable to streams with arbitrary deletion operations. For some applications, the ability to handle 
deletions in a stream may be important. For example, a network monitoring application might be continuously maintaining aggregates over the number of currently open connections per source or destination.

In this paper, we present an algorithm for estimating $F_{k}$, for $k>2$, to within an accuracy of $(1 \pm \epsilon)$ with confidence at least $2 / 3$. (The method can be boosted using the median of averages technique to return high confidence estimates in the standard way $[1,2]$.) The algorithm handles arbitrary insertions and legal deletions (i.e., net frequency of every item is non-negative) from the stream and generalizes the random linear combinations technique of $[1,2]$ designed specifically for estimating $F_{2}$. The space complexity of our method is $\tilde{O}\left(n^{1-\frac{1}{k-1}}\right)$ and the time complexity to process each update is $\tilde{O}(1)$, where, functions of $k$ and $\epsilon$ that do not involve $n$ are treated as constants.

In [7], Coppersmith and Kumar present an algorithm for estimating $F_{k}$ over general update streams. Their algorithm has similar space complexity (i.e., $\tilde{O}\left(n^{1-\frac{1}{k-1}}\right)$ ) as the one we design in this paper. The principal differences between our work and the work in [7] are as follows.

1. Different Technique. Our method constructs random linear combinations of the frequency vector using randomly chosen roots of unity, that is, we construct the sketch $Z=f_{l} x_{l}$, where, $x_{l}$ is a randomly chosen $k^{\text {th }}$ root of unity. Coppersmith and Kumar construct random linear combinations $C=f_{l} x_{l}$, where, for $l \in[N]$, $x_{l}=-1 / n^{1-\frac{1}{k-1}}$ or $1-1 / n^{1-\frac{1}{k-1}}$ with probability $1-1 / n^{1-\frac{1}{k-1}}$ and $1 / n^{1-\frac{1}{k-1}}$ respectively.

2. Symmetric and Simpler Algorithm. Our technique is a symmetric method for all $k \geq 2$, and is a direct generalization of the sketch technique of Alon, Matias and Szegedy [1,2]. In particular, for every $k \geq 2, \mathbf{E}\left[\operatorname{Re} Z^{k}\right]=F_{k}$. The method of Coppersmith and Kumar gives complicated expressions for estimating $F_{k}$, for $k \geq$ 4. For $k=4$, their estimator is $C^{4}-B_{n} F_{2}^{2}$ (where, $B_{n} \approx n^{-4 / 3}\left(1-n^{-2 / 3}\right)^{2}$ ), and requires, in addition, an estimation of $F_{2}$ to within an accuracy factor of $(1 \pm$ $n^{-1 / 3}$ ). The estimator expression for higher values of $k$ (particularly, for powers of 2) are not shown in [7]. These expressions require auxiliary moment estimation and are quite complicated.

3. Time efficient. Our method is significantly more efficient in terms of the time taken to process an arrival over the stream. The time complexity to process a stream update in our method is $\tilde{O}(1)$, whereas, the time complexity of the Coppersmith Kumar technique is $\tilde{O}\left(n^{1-\frac{1}{k-1}}\right)$.

The recent and unpublished work in [11] presents an algorithm for estimating $F_{k}$, for $k>2$ and for the append only streaming model (used by [1,2]), with space complexity $\tilde{O}\left(n^{1-\frac{2}{k+1}}\right)$. Although, the algorithm in [11] improves on the asymptotic space complexity of the algorithm presented in this paper, it cannot handle deletion operations over the stream. Further, the method used by [11] is significantly different from the techniques used in this paper, or from the techniques used by Coppersmith and Kumar [7].

Lower bounds. The work in $[1,2]$ shows space lower bounds for this problem to be $\Omega\left(n^{1-5 / k}\right)$, for any $k>5$. Subsequently, the space lower bounds have been strength- 
ened to $\Omega\left(\epsilon^{2} n^{1-(2+\epsilon) / k}\right)$, for $k>2, \epsilon>0$, by Bar-Yossef, Jayram, Kumar and Sivakumar [3], and further to $\Omega\left(n^{1-2 / k}\right)$ by Chakrabarti, Khot and Sun [5]. Saks and Sun [14] show that estimating the $L_{p}$ distance $d$ between two streaming vectors to within a factor of $d^{\delta}$ requires space $\Omega\left(n^{1-2 / p-4 \delta}\right)$.

Other Related Work. For the special case of computing $F_{2},[1,2]$ presents an $O(\log n+$ $\log m$ ) space and time complexity algorithm, where, $m$ is the sum of the frequencies. Random linear combinations based on random variables drawn from stable distributions were considered by [13] to estimate $F_{p}$, for $0<p \leq 2$. The work presented in [9] presents a sketch technique to estimate the difference between two streams based on the $L_{1}$ metric norm. There has been substantial work on the problem of estimating $F_{0}$ and related metrics (set expression cardinalities over streams) for the various models of data streams $[10,1,4,12]$.

The rest of the paper is organized as follows. Section 2 describes the method and Section 3 presents formal lemmas and their proofs. Finally we conclude in Section 4.

\section{An overview of the method}

In this section, we present a simple description of the algorithm and some of its properties. The lemmas and theorems stated in this section are proved formally in Section 3. Throughout the paper, we treat $k$ as a fixed given value larger than 1 .

\subsection{Sketches using random linear combinations of $k^{\text {th }}$ roots of unity}

Let $x$ be a randomly chosen root of the equation $x^{k}=1$, such that each of the $k$ roots is chosen with equal probability of $1 / k$. Given a complex number $z$, its conjugate is denoted by $\bar{z}$. For any $j, 1 \leq j \leq k$, the following basic property holds, as shown below.

$$
\mathbf{E}\left[x^{j}\right]=\mathbf{E}\left[\bar{x}^{j}\right]= \begin{cases}0 & \text { if } 1 \leq j<k \\ 1 & \text { if } j=k\end{cases}
$$

Proof. Let $j=k$. Then, $\mathbf{E}\left[x^{j}\right]=\mathbf{E}\left[x^{k}\right]=\mathbf{E}[1]=1$, since, $x$ is a root of unity.

Let $1 \leq j<k$ and let $u$ be the elementary $k^{t h}$ root of unity, that is, $u=e^{2 \pi \sqrt{-1} / k}$.

$$
\mathbf{E}\left[x^{j}\right]=\frac{1}{k} \sum_{l=1}^{k}\left(u^{l}\right)^{j}=\frac{1}{k} \sum_{l=1}^{k}\left(u^{j}\right)^{l}=\frac{u^{j}}{k} \frac{\left(1-u^{j k}\right)}{\left(1-u^{j}\right)}
$$

where, the last equality follows from the sum of a geometric progression in the complex field. Since $u^{k}=1$, it follows that $u^{j k}=1$. Further, since $u$ is the elementary $k^{t h}$ root of unity, $u^{j}=e^{2 \pi j \sqrt{-1} / k} \neq 1$, for $1 \leq j<k$. Thus, the expression $\left(1-u^{j k}\right) /(1-$ $\left.u^{j}\right)=0$. Therefore, $\mathbf{E}\left[x^{j}\right]=0$, for $1 \leq j<k$.

The conjugation operator is a 1-1 and onto operator in the field of complex numbers. Further, if $x$ is a root of $x^{k}=1$, then, $\bar{x}^{k}=\overline{x^{k}}=\overline{1}=1$, and therefore, $\bar{x}$ is also a $k^{t h}$ root of unity. Thus, the conjugation operator, applied to the group of $k^{t h}$ roots of unity, results in a permutation of the elements in the group (actually, it is an isomorphism). It 
therefore follows that the sum of the $j^{t h}$ powers of the roots of unity is equal to the sum of the $j^{t h}$ powers of the conjugates of the roots of unity. Thus, $\mathbf{E}\left[\bar{x}^{j}\right]=\mathbf{E}\left[x^{j}\right]$.

Let $Z$ be the random variable defined as $Z=\sum_{l \in[N]} f_{l} x_{l}$. The variable $x_{l}$, for each $l \in[N]$, is one of a randomly chosen root of $x^{k}=1$. The family of variables $\left\{x_{l}\right\}$ is assumed to be $2 k$-wise independent. The following lemma shows that $\operatorname{Re} Z^{k}$ is an unbiased estimator of $F_{k}$. Following [1,2], we call $Z$ as a sketch. The random variable $Z$ can be efficiently maintained with respect to stream updates as follows. First, we choose a random hash function $\theta:[N] \rightarrow[k]$ drawn from a family of hash functions that is $2 k$ wise independent. Further, we pre-compute the $k^{t h}$ roots of unity into an array $A[1 . . k]$ of size $k$ (of complex numbers), that is, $A[r]=e^{2 \cdot \pi \cdot r \cdot \sqrt{-1} / k}$, for $r=1,2, \ldots, k$. For every stream update $(l, v)$, we update the sketch as follows.

$$
Z=Z+v \cdot A[\theta(l)]
$$

The space required to maintain the hash function $\theta=\tilde{O}(k)$, and the time required for processing a stream update is also $\tilde{O}(k)$.

Lemma 1. $\mathbf{E}\left[\operatorname{Re} Z^{k}\right]=F_{k}$.

As the following lemma shows, the variance of this estimator is quite high.

Lemma 2. $\operatorname{Var}\left[\operatorname{Re} Z^{k}\right]=O\left(k^{2 k} F_{2}^{k}\right)$.

This implies that $\operatorname{Var}\left[\operatorname{Re} Z^{k}\right] /\left(\mathbf{E}\left[\operatorname{Re} Z^{k}\right]\right)^{2}=O\left(F_{2}^{k} / F_{k}^{2}\right)$, which could be as large as $n^{k-2}$. To reduce the variance we organize the sketches in a hash table.

\subsection{Organizing sketches in a hash table}

Let $\phi:\{0,1, \ldots, N-1\} \rightarrow[B]$ be a hash function that maps the domain $\{0,1, \ldots, N-$ $1\}$ into a hash table consisting of $B$ buckets. The hash function $\phi$ is drawn from a family of hash functions $\mathcal{H}$ that is $2 k$-wise independent. The random bits used by the hash family is independent of the random bits used by the family $\left\{x_{l}\right\}_{l \in\{0,1, \ldots, N-1\}}$, or, equivalently, the random bits used to generate $\phi$ and $\theta$ are independent. The indicator variable $y_{l, b}$, for any domain element $\left.l \in\{0,1, \ldots, N-1\}\right]$ and bucket $b \in[B]$, is defined as $y_{l, b}=1$ if $\phi(l)=b$ and $y_{l, b}=0$ otherwise. Associated with each bucket $b$ is a sketch $Z_{b}$ of the elements that have hashed to that bucket. The random variables, $Y_{b}$ and $Z_{b}$ are defined as follows.

$$
Z_{b}=\sum_{l} f_{l} \cdot x_{l} \cdot y_{l, b}, \quad Y_{b}=\operatorname{Re} Z_{b}^{k}, \quad \text { and } \quad Y=\sum_{b \in[B]} Y_{b}
$$

Maintaining the hash table of sketches in the presence of stream updates is analogous to maintaining $Z$. As discussed previously, let $\theta:\{0,1, \ldots, N-1\} \rightarrow[k]$ denote a random hash function that is chosen from a $2 k$-wise independent family of hash functions (and independently of the bits used by $\phi$ ), and let $A[1 \ldots k]$ be an array whose $j^{t h}$ entry is $e^{2 \cdot \pi \cdot j \cdot \sqrt{-1} / k}$, for $j=1, \ldots, k$. For every stream update $(l, v)$, we perform the following operation.

$$
Z_{\phi(l)}=Z_{\phi(l)}+v \cdot A[\theta(l)]
$$


The time complexity of the update operation is $\tilde{O}(k)$. The sketches in the buckets except the bucket numbered $\phi(l)$ are left unchanged.

The main observation of the paper is that the hash partitioning of the sketch $Y$ into $\left\{Y_{b}\right\}_{b \in[B]}$ reduces the variance of $Y$ significantly, while maintaining that $\mathbf{E}[Y]=F_{k}$. This is stated in the lemma below.

Lemma 3. Let $B \leq 2 n^{1-\frac{1}{k}}$. Then, $\operatorname{Var}[Y]=O\left(F_{k}^{2} n^{k-2} / B^{k-1}\right)$.

A hash table organization of the sketches is normally used to reduce the time complexity of processing each stream update [6,8]. However, for $k>2$, the hash table organization of the sketches has the additional effect of reducing the variance.

Finally, we keep $s_{1}$ independent copies $Y[0], \ldots, Y\left[s_{1}-1\right]$ of the variable $Y$. The average of these variables is denoted by $\bar{Y}$; thus $\operatorname{Var}[\bar{Y}]=\left(1 / s_{1}\right) \operatorname{Var}[Y]$. The result of the paper is summarized below, which states that $\bar{Y}$ estimates $F_{k}$ to within an accuracy factor of $(1 \pm \epsilon)$ with constant probability greater than $1 / 2$ (at least 2/3).

Theorem 4. Let $n^{1-\frac{1}{k-1}} \leq B \leq 2 \cdot n^{1-\frac{1}{k-1}}$ and $s_{1}=6 \cdot 2^{k} \cdot k^{3 k} / \epsilon^{2}$. Then, $\operatorname{Pr}\left\{\left|\bar{Y}-F_{k}\right|>\epsilon F_{k}\right\} \leq 1 / 3$.

The space usage of the algorithm is therefore $\tilde{O}\left(B \cdot s_{1}\right)=O\left(n^{1-\frac{1}{k-1}}\right)$ bits, since a logarithmic overhead is required to store each sketch $Z_{b}$. To boost the confidence of the answer to at least $1-2^{-\Omega\left(s_{2}\right)}$, a standard technique of returning the median value among $s_{2}$ such average estimates can be used, as shown in $[1,2]$.

The algorithm assumes that the number of buckets in the hash table is $B$, where, $n^{1-\frac{1}{k-1}} \leq B \leq 2 \cdot n^{1-\frac{1}{k-1}}$. Since, in general, the number of distinct items in the stream is not known in advance, one possible method that can be used is as follows. First estimate $n$ to within a factor of $\left(1 \pm \frac{1}{8}\right)$ using an algorithm for estimating $F_{0}$, such as $[10,1,2,4]$. This can be done with high probability, in space $O(\log N)$. Keep $2 \log N+4$ group of (independent) hash tables, such that the $i^{\text {th }}$ group uses $B_{i}=\left\lceil 2^{i / 2}\right\rceil$ buckets. Each group of the hash tables uses the data structure described earlier. At the time of inference, first $n$ is estimated as $\hat{n}$, and, then, we choose a hash table group indexed by $i$ such that $i=2 \cdot\left\lceil\left(1-\frac{1}{k-1}\right) \log (8 \cdot \hat{n} / 7)\right\rceil$. This ensures that the hash table size $B_{i}$ satisfies $n^{1-\frac{1}{k-1}} \leq B_{i} \leq 2 \cdot n^{1-\frac{1}{k-1}}$, with high probability. Since, the number of hash table groups is $2 \cdot \log N$, this construction adds an overhead in terms of both space complexity and update time complexity by a factor of $2 \cdot \log N$. In the remainder of the paper, we assume that $n$ is known exactly, with the understanding that this assumption can be alleviated as described.

\section{Analysis}

The $j^{\text {th }}$ frequency moment of the set of elements that map to bucket $b$ under the hash function $\phi$, is a random variable denoted by $F_{j, b}$. Thus, $F_{j, b}=\sum_{l} f_{l}^{j} y_{l, b}$. Further, since every element in the stream hashes to exactly one bucket, $\sum_{b} F_{j, b}=F_{j}$. We define $h_{l, b}$, for $l \in\{0,1, \ldots, N-1\}$ and $b \in[B]$ to be $h_{l, b}=f_{l} \cdot y_{l, b}$. Thus, $F_{j, b}=\sum_{l} h_{l, b}^{j}$, for $j \geq 1$. 
Notation: Marginal expectations. The random variables, $Y,\left\{Y_{b}\right\}_{b \in B}$ are functions of two families of random variables, namely, $\mathbf{x}=\left\{x_{l}\right\}_{l \in\{0,1, \ldots, N-1\}}$, used to generate the random roots of unity, and $\mathbf{y}=\left\{y_{l, b}\right\}, l \in\{0,1, \ldots, N-1\}$ and $b \in[B]$, used to map elements to buckets in the hash table. Our independence assumptions imply that these two families are mutually independent (i.e., their seeds use independent random bits), that is, $\operatorname{Pr}\{\mathbf{x}=\mathbf{u}$ and $\mathbf{y}=\mathbf{v}\}=\operatorname{Pr}\{\mathbf{x}=\mathbf{u}\} \cdot \operatorname{Pr}\{\mathbf{y}=\mathbf{v}\}$ Let $W=W(\mathbf{x}, \mathbf{y})$ be a random variable that is a function of the random variables in $\mathbf{x}$ and $\mathbf{y}$. For a fixed random choice of $\mathbf{y}=\mathbf{y}_{\mathbf{0}}, \mathbf{E}_{\mathbf{x}}[W]$ denotes the marginal expectation of $W$ as a function of $y$. That is, $\mathbf{E}_{\mathbf{x}}[W]=\sum_{\mathbf{u}} W\left(\mathbf{u}, \mathbf{y}_{\mathbf{0}}\right) \operatorname{Pr}\{\mathbf{x}=\mathbf{u}\}$. It follows that $\mathbf{E}[W]=\mathbf{E}_{\mathbf{y}}\left[\mathbf{E}_{\mathbf{x}}[W]\right]$.

Overview of the analysis. The main steps in the proof of Theorem 4 are as follows. In Section 3.1, we show that $\mathbf{E}_{\mathbf{x}}[Y]=F_{k}$. In Section 3.2, we show that $\mathbf{E}\left[\operatorname{Re} Z^{k}\right] \leq$ $k^{2 k} F_{2}^{k}$. In Section 3.3, using the above result, we show that $\mathbf{E}_{\mathbf{x}}\left[Y^{2}\right] \leq k^{2 k} \sum_{b} F_{2, b}^{k}$. Section 3.4 shows that $\mathbf{E}_{\mathbf{y}}\left[F_{2, b}^{k}\right] \leq\left(2 / B+2^{k} \cdot n^{k-2} / B^{k}\right) F_{k}^{2}$ and also concludes the proof of Theorem 4. Finally, we conclude in Section 4.

Notation: Multinomial Expansion. Let $X$ be defined as $X=\sum_{l \in\{0,1, \ldots, N-1\}} a_{l}$, where, $a_{l} \geq 0$, for $l \in\{0,1, \ldots, N-1\}$. Then, $X^{k}$ can be written as

$$
X^{k}=\sum_{s=1}^{k} \sum_{e_{1}+\cdots e_{s}=k, e_{1}>0, \cdots, e_{s}>0}\left(\begin{array}{c}
k \\
e_{1} e_{2} \cdots e_{s}
\end{array}\right) \sum_{l_{1}<l_{2}<\cdots<l_{s}} a_{l_{1}}^{e_{1}} a_{l_{2}}^{e_{j}} \cdots a_{l_{s}}^{e_{s}}
$$

where, $s$ is the number of distinct terms in the product and $e_{i}$ is the exponent of the $i^{t h}$ product term. The indices $l_{i}$ are therefore necessarily distinct, $l_{i} \in\{0,1, \ldots, N-$ $1\}, i=1,2, \ldots, s$. For easy reference, the above equation is written and used in the following form.

$$
X^{k}=\sum_{s, \mathbf{e}: Q(\mathbf{e}, s)} C(\mathbf{e}) \sum_{\mathbf{1}: R(\mathbf{e}, 1, s)}\left(\prod_{j=1}^{s} a_{l_{j}}^{e_{j}}\right) .
$$

where, $Q(\mathbf{e}, s) \equiv 1 \leq s \leq k$ and $\mathbf{e}=\left(e_{1}, e_{2}, \ldots, e_{s}\right)$ is $s$-dimensional and $\sum_{j=1}^{s} e_{j}=$ $k ; R(\mathbf{e}, \mathbf{l}, s) \equiv \mathbf{l}=\left(l_{1}, l_{2}, \ldots, l_{s}\right)$ is $s$-dimensional and $0 \leq l_{1}<l_{2}<\cdots<l_{s} \leq$ $N-1$; and the multinomial coefficient $C(\mathbf{e})=\left(\begin{array}{c}k \\ e_{1}, \ldots, e_{s}\end{array}\right)$. In this notation, the following inequality holds .

$$
\sum_{\mathbf{l}: R(\mathbf{e}, \mathbf{l}, s)} \prod_{j=1}^{s} a_{l_{j}}^{e_{j}} \leq \prod_{j=1}^{s}\left(\sum_{l} a_{l}^{e_{j}}\right) .
$$

By setting $n=k$, and $a_{1}=a_{2}=\cdots=a_{k}=1$, we obtain,

$$
k^{k}=\sum_{\mathbf{e}, s} C(\mathbf{e})\left(\begin{array}{l}
k \\
s
\end{array}\right)>\sum_{\mathbf{e}, s} C(\mathbf{e}) .
$$

By squaring the above equation on both sides, we obtain that $k^{2 k}=\left(\sum_{\mathbf{e}, s} C(\mathbf{e})\left(\begin{array}{l}k \\ s\end{array}\right)\right)^{2}>$ $\sum_{\mathbf{e}, s} C^{2}(\mathbf{e})$. We therefore have the following inequalities.

$$
\sum_{\mathbf{e}, s} C(\mathbf{e})<k^{k}, \quad \sum_{\mathbf{e}, s} C^{2}(\mathbf{e})<k^{2 k} .
$$




\subsection{Expectation}

In this section, we show that $\mathbf{E}\left[\operatorname{Re} Z^{k}\right]=F_{k}$, thereby proving Lemma 1 , and that $\mathbf{E}_{\mathbf{x}}[Y]=F_{k}$.

Proof (of Lemma 1). Since the family of variables $x_{l}$ 's is $k$-wise independent, therefore

$$
\mathbf{E}\left[\prod_{j=1}^{s} x_{l_{j}}^{e_{j}}\right]=\prod_{j=1}^{s} \mathbf{E}\left[x_{l_{j}}^{e_{j}}\right] .
$$

Applying equation (2) to $Z^{k}=\left(\sum_{l} f_{l} x_{l}\right)^{k}$ and using linearity of expectation and $k$ wise independence property of $x_{l}$ 's, we obtain

$$
\mathbf{E}\left[Z^{k}\right]=\sum_{s, \mathbf{e}: Q(\mathbf{e}, s)} C(\mathbf{e}) \sum_{\mathbf{1}: R(\mathbf{e}, \mathbf{l}, s)}\left(\prod_{j=1}^{s} f_{l_{j}}^{e_{j}}\right)\left(\prod_{j=1}^{s} \mathbf{E}\left[x_{l_{j}}^{e_{j}}\right]\right) .
$$

Using equation (1), we note that the term $\left(\prod_{j=1}^{s} \mathbf{E}\left[x_{l_{j}}^{e_{j}}\right]\right)=0$, if $s>1$, since in this case, $e_{j}<k$, for each $j=1, \ldots, s$. Thus, the above summation reduces to

$$
\mathbf{E}\left[Z^{k}\right]=\sum_{l} f_{l}^{k}=F_{k}
$$

Since $F_{k}$ is real, $\mathbf{E}\left[\operatorname{Re} Z^{k}\right]$ is also $F_{k}$, proving Lemma 1 .

Lemma 5. Suppose that the family of random variables $\left\{x_{l}\right\}$ is $k$-wise independent. Then, $\mathbf{E}_{\mathbf{x}}\left[Y_{b}\right]=F_{k, b}$ and $\mathbf{E}_{\mathbf{x}}[Y]=\mathbf{E}[Y]=F_{k}$.

Proof. We first show that $\mathbf{E}_{\mathbf{x}}\left[Y_{b}\right]=F_{k, b} . \mathbf{E}_{\mathbf{x}}\left[Z_{b}^{k}\right]=\mathbf{E}_{\mathbf{x}}\left[\left(\sum_{l} f_{l} y_{l, b} x_{l}\right)^{k}\right]=\mathbf{E}_{\mathbf{x}}\left[\left(\sum_{l} h_{l, b} x_{l}\right)^{k}\right]$, by letting $h_{l, b}=f_{l} \cdot y_{l, b}$. By an argument analogous to the proof of Lemma 1, we obtain $\mathbf{E}_{\mathbf{x}}\left[\left(\sum_{l} h_{l, b} x_{l}\right)^{k}\right]=\sum_{l} h_{l, b}^{k}=\sum_{l} f_{l}^{k} y_{l, b}^{k}=\sum_{l} f_{l, k}^{k} y_{l, b}=F_{k, b}$, (since $y_{l, b}$ 's are binary variables). Since $F_{k, b}$ is always real, $\mathbf{E}_{\mathbf{x}}\left[Y_{b}\right]=\mathbf{E}_{\mathbf{x}}\left[\operatorname{Re} Z_{b}^{k}\right]=F_{k, b}$. Finally, $\mathbf{E}_{\mathbf{x}}[Y]=\mathbf{E}_{\mathbf{x}}\left[\sum_{b} Y_{b}\right]=\sum_{b} \mathbf{E}_{\mathbf{x}}\left[Y_{b}\right]=\sum_{b} F_{k, b}=F_{k}$, since each element is hashed to exactly one bucket. Further, $\mathbf{E}[Y]=\mathbf{E}_{\mathbf{y}}\left[\mathbf{E}_{\mathbf{x}}[Y]\right]=\mathbf{E}_{\mathbf{y}}\left[F_{k}\right]=F_{k}$.

\subsection{Variance of $\operatorname{Re} Z^{k}$}

In this section, we estimate the variance of $\operatorname{Re} Z^{k}$ and derive some simple corollaries.

Lemma 6. Let $W=\operatorname{Re}\left(\sum_{l} a_{l} x_{l}\right)^{k}$. Then, $\operatorname{Var}[W] \leq k^{2 k}\left(\sum_{l} a_{l}^{2}\right)^{k}$.

Proof. Let $X=\left(\sum_{l} a_{l} x_{l}\right)^{k}$. Then, $\operatorname{Var}[W]=\mathbf{E}\left[W^{2}\right]-(\mathbf{E}[W])^{2} \leq \mathbf{E}[X \bar{X}]-$ $(\mathbf{E}[W])^{2}$. Using equation (2), for $X, \bar{X}$, we obtain the following.

$$
\begin{aligned}
& X=\sum_{s, \mathbf{e}: Q(\mathbf{e}, s)} C(\mathbf{e}) \sum_{\mathbf{1}: R(\mathbf{e}, \mathbf{l}, s)}\left(\prod_{j=1}^{s} a_{l_{j}}^{e_{j}}\right) \cdot\left(\prod_{j=1}^{s} x_{l_{j}}^{e_{j}}\right) \\
& \bar{X}=\sum_{t, \mathbf{g}: Q(\mathbf{g}, t)} C(\mathbf{g}) \sum_{\mathbf{l}: R(\mathbf{g}, \mathbf{m}, t)}\left(\prod_{j^{\prime}=1}^{t} a_{m_{j^{\prime}}}^{g_{j^{\prime}}}\right) \cdot\left(\prod_{j^{\prime}=1}^{t} \bar{x}_{m_{j^{\prime}}}^{g_{j^{\prime}}}\right)
\end{aligned}
$$


Multiplying the above two equations, we obtain

$$
\begin{aligned}
& X \cdot \bar{X}= \sum_{s, \mathbf{e}: Q(\mathbf{e}, s)} \sum_{t, \mathbf{g}: Q(\mathbf{g}, t)} C(\mathbf{e}) \cdot C(\mathbf{g}) \sum_{\mathbf{l}: R(\mathbf{e}, \mathbf{l}, s)} \sum_{\mathbf{l}: R(\mathbf{g}, \mathbf{m}, t)} \\
&\left(\prod_{j=1}^{s} a_{l_{j}}^{e_{j}}\right) \cdot\left(\prod_{j^{\prime}=1}^{t} a_{m_{j^{\prime}}}^{g_{j^{\prime}}}\right) \cdot\left(\prod_{j=1}^{s} x_{l_{j}}^{e_{j}}\right) \cdot\left(\prod_{j^{\prime}=1}^{t} \bar{x}_{m_{j^{\prime}}}^{g_{j^{\prime}}}\right) .
\end{aligned}
$$

The general form of the product of random variables that arises in the multinomial expansion of $X \bar{X}$ is $\left(\prod_{j=1}^{s} x_{l_{j}}^{e_{j}}\right)\left(\prod_{j^{\prime}=1}^{t} \bar{x}_{m_{j^{\prime}}}^{g_{j^{\prime}}}\right)$. Since the random variables $x_{l}$ 's are $2 k$ wise independent, using equation (1), it follows that,

$$
\mathbf{E}\left[\prod_{j=1}^{s} x_{l_{j}}^{e_{j}} \prod_{j^{\prime}=1}^{t} \bar{x}_{m_{j^{\prime}}}^{g_{j}}\right]= \begin{cases}1 & \text { if } s=t=1, e_{1}=g_{1}=k \\ 1 & \text { if } s=t, t>1, \mathbf{e}=\mathbf{g} \text { and } \mathbf{l}=\mathbf{m} \\ 0 & \text { otherwise }\end{cases}
$$

This directly yields the following.

$$
\begin{aligned}
\mathbf{E}[X \bar{X}] & =\sum_{\mathbf{e}, s: Q(\mathbf{e}, s)} C^{2}(\mathbf{e}) \sum_{\mathbf{l}: R(\mathbf{e}, \mathbf{l}, s)} \prod_{j=1}^{s} a_{l_{j}}^{2 e_{j}} \\
& \leq \sum_{\mathbf{e}, s} C^{2}(\mathbf{e}) \prod_{j=1}^{s}\left(\sum_{l} a_{l}^{2 e_{j}}\right), \text { by equation (3) } \\
& \leq \sum_{\mathbf{e}, s} C^{2}(\mathbf{e}) \prod_{j=1}^{s}\left(\sum_{l} a_{l}^{2}\right)^{e_{j}}, \text { since } \sum_{l} a_{l}^{2 e_{j}} \leq\left(\sum_{l} a_{l}^{2}\right)^{e_{j}} \\
& =\left(\sum_{l} a_{l}^{2}\right)^{k}\left(\sum_{\mathbf{e}, s} C^{2}(\mathbf{e})\right), \quad \text { since } \sum_{j=1}^{s} e_{j}=k \\
& \leq\left(\sum_{l} a_{l}^{2}\right)^{k} \cdot k^{2 k}, \quad \text { by equation (4). }
\end{aligned}
$$

By letting $a_{l}=f_{l}, l \in\{0,1,2 \ldots, N-1\}$, Lemma 6 yields

$$
\operatorname{Var}\left[\operatorname{Re} Z^{k}\right]=\operatorname{Var}\left[\operatorname{Re}\left(\sum_{l} f_{l} x_{l}\right)^{k}\right] \leq k^{2 k} F_{2}^{k},
$$

which is the statement of Lemma 2. By letting $a_{l}=h_{l, b}=f_{l} \cdot y_{l, b}$, where, $b$ is a fixed bucket index, and $l \in\{0,1,2 \ldots, N-1\}$, yields the following equation.

$$
\mathbf{E}_{\mathbf{x}}\left[Y_{b}^{2}\right] \leq k^{2 k} F_{2, b}^{k}, \quad \text { for } b \in[B] .
$$

\section{3 $\operatorname{Var}[Y]$ : Vanishing of cross-bucket terms}

We now consider the problem of obtaining an upper bound on $\operatorname{Var}[Y]$. Note that $\operatorname{Var}[Y]=\mathbf{E}_{\mathbf{y}}\left[\mathbf{E}_{\mathbf{x}}\left[Y^{2}\right]\right]-\left(\mathbf{E}_{\mathbf{y}}\left[\mathbf{E}_{\mathbf{x}}[Y]\right]\right)^{2}$. From Lemma 5, $\mathbf{E}_{\mathbf{y}}\left[\mathbf{E}_{\mathbf{x}}[Y]\right]=F_{k}$. Thus,

$$
\operatorname{Var}[Y]=\mathbf{E}_{\mathbf{y}}\left[\mathbf{E}_{\mathbf{x}}\left[Y^{2}\right]\right]-F_{k}^{2}
$$


Lemma 7. $\operatorname{Var}[Y] \leq k^{2 k} \sum_{b} \mathbf{E}_{\mathbf{y}}\left[F_{2, b}^{k}\right]$, assuming independence assumption I.

Proof. $\mathbf{E}_{\mathbf{x}}\left[Y^{2}\right]=\mathbf{E}_{\mathbf{x}}\left[\left(\sum_{b} Y_{b}\right)^{2}\right]=\mathbf{E}_{\mathbf{x}}\left[\sum_{b} Y_{b}^{2}+\sum_{a \neq b} Y_{a} Y_{b}\right]=\sum_{b} \mathbf{E}_{\mathbf{x}}\left[Y_{b}^{2}\right]+$ $\sum_{a \neq b} \mathbf{E}_{\mathbf{x}}\left[Y_{a} Y_{b}\right]$.

We now consider $\mathbf{E}_{\mathbf{x}}\left[Y_{a} Y_{b}\right]$, for $a \neq b$. Recall that $Y_{a}=\operatorname{Re} Z_{a}^{k}$ ( and analogously, $Y_{b}$ is defined). For any two complex numbers $z, w,(\operatorname{Re} z)(\operatorname{Re} w)=(1 / 2) \operatorname{Re}(z(w+$ $\bar{w})$ ). Thus, $Y_{a} Y_{b}=\left(\operatorname{Re} Z_{a}^{k}\right)\left(\operatorname{Re} Z_{b}^{k}\right)=(1 / 2) \operatorname{Re}\left(Z_{a}^{k} Z_{b}^{k}+Z_{a}^{k} \overline{Z_{b}^{k}}\right)$.

Let us first consider $\mathbf{E}_{\mathbf{x}}\left[Z_{a}^{k} Z_{b}^{k}\right]$. The general term involving product of random variables is $\left(\prod_{j=1}^{s} f_{l_{j}}^{e_{j}}\right) \cdot\left(\prod_{j^{\prime}=1}^{t} f_{m_{j}}^{g_{j^{\prime}}}\right) \cdot\left(\prod_{j=1}^{s} y_{l_{j}, a} \cdot x_{l_{j}}^{e_{j}}\right) \cdot\left(\prod_{j^{\prime}=1}^{t} y_{m_{j^{\prime}}, b} \cdot x_{m_{j^{\prime}}}^{g_{j}}\right)$. Consider the last two product terms in the above expression, that is, $\left(\prod_{j=1}^{s} y_{l_{j}, a} \cdot x_{l_{j}}^{e_{j}}\right)$. $\left(\prod_{j^{\prime}=1}^{t} y_{m_{j^{\prime}}, b} \cdot x_{m_{j^{\prime}}}^{g_{j}}\right)$. For any $1 \leq j \leq s$ and $1 \leq j^{\prime} \leq t$, it is not possible that $l_{j}=m_{j^{\prime}}$, that is, the same element whose index is given by $l_{j}=m_{j^{\prime}}$ cannot simultaneously hash to two distinct buckets, $a$ and $b$ (recall that $a \neq b$ ). By $2 k$-wise independence, we therefore obtain that the only way the above product term can be non zero (i.e., 1) on expectation, is that $s=t=1$ and therefore, $e_{1}=k$ and $g_{1}=k$. Thus, we have $\mathbf{E}\left[Z_{a}^{k} Z_{b}^{k}\right]=\sum_{l, m} h_{l, a}^{k} h_{m, b}^{k}=F_{k, a} F_{k, b}$.

Using the same observation, it can be argued that $\mathbf{E}_{\mathbf{x}}\left[Z_{a}^{k} \overline{Z_{b}^{k}}\right]=F_{k, a} F_{k, b}$. It follows that $\left.\mathbf{E}_{\mathbf{x}}\left[(1 / 2)\left(Z_{a}^{k} Z_{b}^{k}+Z_{a}^{k} \overline{Z_{b}^{k}}\right)\right)\right]=F_{k, a} F_{k, b}$, which is a real number. Therefore $\mathbf{E}_{\mathbf{x}}\left[\operatorname{Re}(1 / 2)\left(Z_{a}^{k} Z_{b}^{k}+Z_{a}^{k} \overline{Z_{b}^{k}}\right)\right]=F_{k, a} F_{k, b}=\mathbf{E}_{\mathbf{x}}\left[Y_{a} Y_{b}\right]$.

By equation (7), $\operatorname{Var}[Y]=\mathbf{E}_{\mathbf{y}}\left[\mathbf{E}_{\mathbf{x}}\left[Y^{2}\right]\right]-F_{k}^{2}$. Further, from Lemma $5, F_{k}=$ $\mathbf{E}_{\mathbf{y}}\left[\sum_{b} F_{k, b}\right]$. We therefore have,

$$
\begin{aligned}
\operatorname{Var}[Y] & =\mathbf{E}_{\mathbf{y}}\left[\mathbf{E}_{\mathbf{x}}\left[Y^{2}\right]-\left(\sum_{b} F_{k, b}\right)^{2}\right] \\
& =\mathbf{E}_{\mathbf{y}}\left[\sum_{b} \mathbf{E}_{\mathbf{x}}\left[Y_{b}^{2}\right]+\sum_{a \neq b} \mathbf{E}_{\mathbf{x}}\left[Y_{a} Y_{b}\right]-\left(\sum_{b} F_{k, b}\right)^{2}\right] \\
& =\mathbf{E}_{\mathbf{y}}\left[\sum_{b} \mathbf{E}_{\mathbf{x}}\left[Y_{b}^{2}\right]+\sum_{a \neq b} F_{k, a} F_{k, b}-\left(\sum_{b} F_{k, b}\right)^{2}\right], \quad \text { by above argument } \\
& =\mathbf{E}_{\mathbf{y}}\left[\sum_{b} \mathbf{E}_{\mathbf{x}}\left[Y_{b}^{2}\right]-\sum_{b} F_{k, b}^{2}\right] \\
& \leq \mathbf{E}_{\mathbf{y}}\left[\sum_{b} \mathbf{E}_{\mathbf{x}}\left[Y_{b}^{2}\right]\right] \\
& \leq \mathbf{E}_{\mathbf{y}}\left[\sum_{b} k^{2 k} F_{2, b}^{k}\right], \quad \text { by equation (6) }
\end{aligned}
$$

\subsection{Calculation of $\mathrm{E}\left[\mathrm{F}_{2, b}^{k}\right]$}

Given a $t$-dimensional vector $\mathbf{e}=\left(e_{1}, \ldots, e_{t}\right)$ such that $e_{i}>0$, for $1 \leq i \leq t$ and $\sum_{j=1}^{t} e_{j}=k$, we define the function $\psi(\mathbf{e})$ as follows. Without loss of generality, let the indices $e_{j}$ be arranged in non-decreasing order. Let $r=r(\mathbf{e})$ denote the largest index such that $e_{r}<k / 2$. Then, we define the function $\phi(e)$ as follows.

$$
\psi(\mathbf{e})=n^{\sum_{j=1}^{r}\left(1-2 e_{j} / k\right)} / B^{t}
$$


The motivation of this definition stems from its use in the following lemma.

Lemma 8. Suppose $\sum_{j=1}^{t} e_{j}=k$ and $e_{j}>0$, for $j=1, \ldots, t$. Then, $\prod_{j=1}^{t} F_{2 e_{j}} \leq$ $\psi(\mathbf{e}) \cdot F_{k}^{2} \cdot B^{t}$.

Proof. From [1,2], $F_{j} \leq n^{1-j / k} F_{k}^{j / k}$, if $j<k$ and $F_{j} \leq F_{k}^{j / k}$, if $j>k$. Thus,

$$
\begin{aligned}
\prod_{j=1}^{t} F_{2 e_{j}} & =\left(\prod_{j=1}^{r} F_{2 e_{j}}\right)\left(\prod_{j=r+1}^{t} F_{2 e_{j}}\right)=\left(\prod_{j=1}^{r} n^{1-2 e_{j} / k} F_{k}^{2 e_{j} / k}\right)\left(\prod_{j=r+1}^{t} F_{k}^{2 e_{j} / k}\right) \\
& =n^{\sum_{j=1}^{r}\left(1-2 e_{j} / k\right)} F_{k}^{\sum_{j=1}^{t} 2 e_{j} / k}=\psi(\mathbf{e}) \cdot B^{t} \cdot F_{k}^{2}, \quad \text { since } \sum_{j} e_{j}=k .
\end{aligned}
$$

The function $\psi$ satisfies the following property that we use later.

Lemma 9. If $B<2 \cdot n^{1-\frac{1}{k}}$, then, $\psi(\mathbf{e}) \leq \max \left(2 / B, 2^{k} \cdot n^{k-2} / B^{k}\right)$.

Proof. Let e be a $t$-dimensional vector. If $t=1, \psi(\mathbf{e})=1 / B$. If $t=r$, then $\psi(\mathbf{e})=$ $n^{t-2} / B^{t} \leq 2^{k} \cdot n^{k-2} / B^{k}$. If $t \geq r+2$, then $\psi(\mathbf{e})=\left(2^{t} / B^{t}\right) \cdot n^{t-\left((t-r)+\sum 2 e_{j} / k\right)}<$ $2^{t} \cdot n^{t-2} / B^{t} \leq 2^{k} \cdot n^{k-2} / B^{k}$. Finally, let $t=r+1$. Then,

$$
\psi(\mathbf{e})=2^{t} \cdot n^{t-1-2 \sum e_{j} / k} / B^{t} \leq 2^{t} \cdot n^{t-1-2(t-1) / k} / B^{t},
$$

since $\sum_{j=1}^{r} e_{j} \geq r=t-1$. Thus,

$$
\begin{aligned}
& \psi(\mathbf{e}) \leq \psi(\mathbf{e})\left(2 \cdot n^{1-\frac{1}{k}} / B\right)^{k-t} \leq\left(2^{t} \cdot n^{t-1-2(t-1) / k} / B^{t}\right)\left(2 \cdot n^{1-\frac{1}{k}} / B\right)^{k-t}= \\
& 2^{k} \cdot n^{k-2-(t-2) / k} / B^{k} \leq 2^{k} \cdot n^{k-2} / B^{k} .
\end{aligned}
$$

where, the first inequality follows from the assumption that $B<n^{1-\frac{1}{k}}$ and the second inequality follows because $t \geq 2$.

Lemma 10. Let $B<2 \cdot n^{1-\frac{1}{k}}$. Then, $\mathbf{E}\left[F_{2, b}^{k}\right]<k^{k} F_{k}^{2}\left(2 / B+2^{k} \cdot n^{k-2} / B^{k}\right)$.

Proof. For a fixed $b$, the variables $y_{l, b}$ are $k$-wise independent. $F_{2, b}$ is a linear function of $y_{l, b}$. Thus, $F_{2, b}^{k}$ is a symmetric multinomial of degree $k$, as follows.

$$
\begin{aligned}
F_{2, b}^{k} & =\left(\sum_{l} f_{l}^{2} y_{l, b}\right)^{k} \\
& =\sum_{s, \mathbf{e}} C(\mathbf{e}) \sum_{l_{1}<l_{2}<\cdots l_{s}} f_{l_{1}}^{2 e_{1}} \cdots f_{l_{s}}^{2 e_{s}} y_{l_{1}, b} \cdot y_{l_{2}, b} \cdot y_{l_{s}, b} .
\end{aligned}
$$


Taking expectations, and using $k$-wise independence of the $y_{l, b}$ 's, we have,

$$
\begin{aligned}
\mathbf{E}\left[F_{2, b}^{k}\right] & =\sum_{s, \mathbf{e}} C(\mathbf{e}) \sum_{l_{1}<l_{2}<\cdots<l_{s}} f_{l_{1}}^{2 e_{1}} \cdots f_{l_{j}}^{2 e_{j}} \mathbf{E}\left[y_{l_{1}, b} \cdot y_{l_{2}, b} \cdot y_{l_{s}, b}\right] \\
& =\sum_{s, \mathbf{e}} C(\mathbf{e}) \sum_{l_{1}<l_{2}<\cdots<l_{s}} f_{l_{1}}^{2 e_{1}} \cdots f_{l_{j}}^{2 e_{j}} \mathbf{E}\left[y_{l_{1}, b}\right] \cdot \mathbf{E}\left[y_{l_{2}, b}\right] \cdots \mathbf{E}\left[y_{l_{s}}, b\right] \\
& =\sum_{s, \mathbf{e}} C(\mathbf{e}) \sum_{l_{1}<l_{2}<\cdots<l_{s}} f_{l_{1}}^{2 e_{1}} \cdots f_{l_{j}}^{2 e_{j}} \frac{1}{B^{s}}, \quad \text { since, } \mathbf{E}\left[y_{l_{j}, b}\right]=\frac{1}{B} \\
& \leq \sum_{s, \mathbf{e}} C(\mathbf{e}) \cdot\left(1 / B^{s}\right) \cdot \prod_{j=1}^{s} F_{2 e_{j}} \\
& \leq \sum_{s, \mathbf{e}} C(\mathbf{e}) \cdot \psi(\mathbf{e}) \cdot F_{k}^{2}, \quad \text { by Lemma } 8 \\
& \leq \sum_{s, \mathbf{e}} C(\mathbf{e}) \cdot F_{k}^{2} \cdot\left(2 / B+2^{k} \cdot n^{k-2} / B^{k}\right), \quad \text { by Lemma } 9 \\
& \leq k^{k} \cdot F_{k}^{2} \cdot\left(2 / B+2^{k} \cdot n^{k-2} / B^{k}\right), \quad \text { since, } \sum_{s, \mathbf{e}} C(\mathbf{e})<k^{k}
\end{aligned}
$$

Combining the result of Lemma 7 with Lemma 10, we obtain the following bound on $\operatorname{Var}[Y]$.

$$
\operatorname{Var}[Y] \leq k^{3 k} \cdot F_{k}^{2} \cdot\left(2+2^{k} \cdot n^{k-2} / B^{k-1}\right)
$$

Recall that $\bar{Y}$ is the average of $s_{1}$ independent estimators, each calculating $Y$. The main theorem of the paper now follows simply.

Proof (of Theorem 4). By Chebychev's inequality, $\operatorname{Pr}\left\{\left|\bar{Y}-F_{k}\right|>\epsilon F_{k}\right\}<\operatorname{Var}[\bar{Y}] /\left(\epsilon^{2} F_{k}^{2}\right)$. Substituting Equation (8), we have $\operatorname{Var}[\bar{Y}] /\left(\epsilon^{2} \cdot F_{k}^{2}\right) \leq 1 / 3$.

\section{Conclusions}

The paper presents a method for estimating the $k^{t h}$ frequency moment, for $k>2$, of data streams with general update operations. The algorithm has space complexity $\left.\tilde{O}\left(n^{1-\frac{1}{k-1}}\right)\right)$ and is based on constructing random linear combinations using randomly chosen $k^{t h}$ roots of unity. A gap remains between the lower bound for this problem, namely, $O\left(n^{1-2 / k}\right)$, for $k>2$, as proved in [3,5] and the complexity of a known algorithm for this problem.

\section{References}

1. Noga Alon, Yossi Matias, and Mario Szegedy. "The Space Complexity of Approximating the Frequency Moments". In Proceedings of the 28th Annual ACM Symposium on the Theory of Computing STOC, 1996, pages 20-29, Philadelphia, Pennsylvania, May 1996.

2. Noga Alon, Yossi Matias, and Mario Szegedy. "The space complexity of approximating frequency moments”. Journal of Computer Systems and Sciences, 58(1):137-147, 1998. 
3. Ziv Bar-Yossef, T.S. Jayram, Ravi Kumar, and D. Sivakumar. "An information statistics approach to data stream and communication complexity". In Proceedings of the 34th ACM Symposium on Theory of Computing (STOC), 2002, pages 209-218, Princeton, NJ, 2002.

4. Ziv Bar-Yossef, T.S. Jayram, Ravi Kumar, D. Sivakumar, and Luca Trevisan. "Counting distinct elements in a data stream". In Proceedings of the 6th International Workshop on Randomization and Approximation Techniques in Computer Science, RANDOM 2002, Cambridge, MA, 2002.

5. Amit Chakrabarti, Subhash Khot, and Xiaodong Sun. "Near-Optimal Lower Bounds on the Multi-Party Communication Complexity of Set Disjointness". In Proceedings of the 18th Annual IEEE Conference on Computational Complexity, CCC 2003, Aarhus, Denmark, 2003.

6. Moses Charikar, Kevin Chen, and Martin Farach-Colton. "Finding frequent items in data streams". In Proceedings of the 29th International Colloquium on Automata Languages and Programming, 2002.

7. Don Coppersmith and Ravi Kumar. "An improved data stream algorithm for estimating frequency moments". In Proceedings of the Fifteenth ACM SIAM Symposium on Discrete Algorithms, New Orleans, LA, 2004.

8. G. Cormode and S. Muthukrishnan. "What's Hot and What's Not: Tracking Most Frequent Items Dynamically". In Proceedings of the Twentysecond ACM SIGACT-SIGMOD-SIGART Symposium on Principles of Database Systems, San Diego, California, May 2003.

9. Joan Feigenbaum, Sampath Kannan, Martin Strauss, and Mahesh Viswanathan. "An Approximate $L^{1}$-Difference Algorithm for Massive Data Streams". In Proceedings of the 40th Annual IEEE Symposium on Foundations of Computer Science, New York, NY, October 1999.

10. Philippe Flajolet and G.N. Martin. "Probabilistic Counting Algorithms for Database Applications". Journal of Computer Systems and Sciences, 31(2):182-209, 1985.

11. Sumit Ganguly. "A bifocal technique for estimating frequency moments over data streams". Manuscript, April 2004.

12. Sumit Ganguly, Minos Garofalakis, and Rajeev Rastogi. "Processing Set Expressions over Continuous Update Streams". In Proceedings of the 2003 ACM SIGMOD International Conference on Management of Data, San Diego, CA, 2003.

13. Piotr Indyk. "Stable Distributions, Pseudo Random Generators, Embeddings and Data Stream Computation". In Proceedings of the 41st Annual IEEE Symposium on Foundations of Computer Science, pages 189-197, Redondo Beach, CA, November 2000.

14. M. Saks and X. Sun. "Space lower bounds for distance approximation in the data stream model". In Proceedings of the 34th ACM Symposium on Theory of Computing (STOC), 2002, 2002. 